\title{
Geodesic spaces of low Nagata dimension
}

\author{
MARTina JøRGensen and URs LANG
}

\begin{abstract}
We show that every geodesic metric space admitting an injective continuous map into the plane as well as every planar graph has Nagata dimension at most two, hence asymptotic dimension at most two. This relies on and answers a question in a recent work by Fujiwara and Papasoglu. We conclude that all three-dimensional Hadamard manifolds have Nagata dimension three. As a consequence, all such manifolds are absolute Lipschitz retracts.
\end{abstract}

\section{Nagatan mielessä matalaulotteisia geodeettisia avaruuksia}

Tiivistelmä. Osoitamme, että jokaisen tasoverkon ja jokaisen injektiivisesti ja jatkuvasti tasoon uppoavan geodeettisen metrisen avaruuden Nagatan ulottuvuus, ja täten asymptoottinen ulottuvuus, on korkeintaan kaksi. Tulos perustuu ja vastaa Fujiwaran ja Papasoglun viimeaikaisessa työssä esitettyyn kysymykseen. Johdamme tästä, että jokaisen kolmiulotteisen Hadamardin moniston Nagatan ulottuvuus on kolme, ja täten jokainen tällainen monisto on ehdoton Lipschitzin vetäytymä.

\section{Introduction}

Gromov's notion of asymptotic dimension has become an indispensable tool in the investigation of large scale properties of metric spaces and infinite groups. In the recent paper [10], Fujiwara and Papasoglu show that all planar geodesic metric spaces and planar graphs have asymptotic dimension at most three. Here, a geodesic metric space is said to be planar if it admits an injective continuous map into $\mathbb{R}^{2}$, and a not necessarily locally finite (but connected) graph, viewed as a geodesic metric space with edges of length one, is called planar if it admits an injective map into $\mathbb{R}^{2}$ whose restriction to every edge, and hence every finite subgraph, is continuous. In fact, Fujiwara and Papasoglu prove the stronger result that the (Assouad-)Nagata dimension, which in general is greater than or equal to the asymptotic dimension, is at most 3 (see Sect. 2 for the definitions of these notions). This is achieved by showing that for some universal constant $C$, every metric annulus of width comparable to $s>0$ admits a covering by subsets of diameter at most $C s$ such that every $s$-ball meets no more than two of them. For an appropriate sequence of annuli covering the underlying space $X$, the union of the individual covers has $s$-multiplicity at most 4 . This yields the bound on the dimension, which exceeds the expected value by one.

In this note, we first observe that the bound on the Nagata dimension can be improved to 2 (see Theorem 2). This answers in particular Question 5.1 in [10] for the asymptotic dimension. For the proof, we combine the above result for metric annuli with a simplified version of the Hurewicz-type theorem from [8] for the case of a Lipschitz function $f: X \rightarrow \mathbb{R}$ (see Theorem 1). The latter holds in all dimensions, and we provide a streamlined argument. We then take a further step and apply

https://doi.org/10.54330/afm.112472

2020 Mathematics Subject Classification: Primary 53C23, 53C21, 54F45.

Key words: Asymptotic dimension, Assouad-Nagata dimension, planar graph, Hadamard manifold, absolute Lipschitz retract.

(c) 2022 The Finnish Mathematical Society 
Theorem 2 and Theorem 1 to prove that all 3-dimensional Hadamard manifolds have Nagata dimension 3 (see Theorem 3). The two-dimensional analogue was shown in Theorem 5.7.3 in [17], but in higher dimensions, previous results depend on additional curvature bounds or homogeneity assumptions (compare [11, 16]). Finiteness of the asymptotic dimension or the Nagata dimension has a number of important consequences (see [4] for a survey). It now follows from Theorem 3 and a general criterion established in [16] that all 3-dimensional Hadamard manifolds are absolute Lipschitz retracts (see Theorem 4). Again, this subsumes some earlier results with extra conditions (compare $[9,15])$.

After the posting of the first version [14] of the present paper, another proof of Theorem 2 based on [10] and [8] appeared in the preprint [6], which was later superseded by [7]. This work discusses a number of further results pertaining to non-planar graphs and surfaces.

\section{Definitions}

We now state the relevant definitions. Let $(X, d)$ be a metric space. A collection $\mathscr{C}$ of subsets of $X$ is called $D$-bounded, for some constant $D$, if every set $C \in \mathscr{C}$ has diameter

$$
\operatorname{diam}(C):=\sup \{d(x, y): x, y \in C\} \leq D .
$$

Given $s>0$, the $s$-multiplicity of $\mathscr{C}$ is the infimum of all integers $m \geq 0$ such that every closed $s$-ball in $X$ meets at most $m$ members of the collection. The asymptotic dimension $\operatorname{asdim}(X)$ of $X$, a quasi-isometry and coarse invariant, is the infimum of all integers $n \geq 0$ for which there is a function $D:(0, \infty) \rightarrow(0, \infty)$ such that for every $s>0, X$ possesses a $D(s)$-bounded covering with $s$-multiplicity at most $n+1$ [11]. The Nagata dimension or Assouad-Nagata dimension $\operatorname{dim}_{\mathrm{N}}(X)$ of $X$, a bi-Lipschitz and quasi-symmetry invariant, is defined analogously with a linear control function $D(s)=c s[1,16]$. To make the constant $c>0$ explicit, we say that $X$ has Nagata dimension $n$ or at most $n$ with constant $c$. It is an essential feature of the results in [10] and those obtained here that they hold uniformly for all members of the class of spaces considered, that is, with the same constant $c$.

The following equivalent formulation is often useful. Let $\mathscr{C}$ be a collection of subsets of $X$. For $s>0, \mathscr{C}$ is called $s$-disjoint if

$$
d\left(C, C^{\prime}\right):=\inf \left\{d\left(x, x^{\prime}\right): x \in C, x^{\prime} \in C^{\prime}\right\} \geq s
$$

whenever $C, C^{\prime} \in \mathscr{C}$ are distinct. More generally, we say that $\mathscr{C}$ is $(n+1, s)$-disjoint if $\mathscr{C}=\bigcup_{i=1}^{n+1} \mathscr{C}_{i}$ for subcollections $\mathscr{C}_{i}$ that are individually $s$-disjoint. We think of the indices $1, \ldots, n+1$ as colours of $\mathscr{C}$. Evidently, if $\mathscr{C}$ is $(n+1, s)$-disjoint, then $\mathscr{C}$ has $\lambda s$-multiplicity at most $n+1$ for any $\lambda \in\left(0, \frac{1}{2}\right)$. Hence, a metric space $X$ with an $(n+1, s)$-disjoint and $c^{\prime} s$-bounded cover for every $s>0$ satisfies $\operatorname{dim}_{N}(X) \leq n$ with any constant $c>2 c^{\prime}$. Conversely, the following holds (see the proof of Proposition 2.5 in $[16])$.

Proposition 1. If $X$ is a metric space with a cs-bounded cover of s-multiplicity at most $n+1$ for some $c, s>0$, then $X$ also admits an $(n+1, \lambda s)$-disjoint and $c^{\prime} \lambda s$-bounded cover, where $\lambda, c^{\prime}>0$ depend only on $c$ and $n$. In particular, if $X$ has Nagata dimension at most $n$ with constant $c$, then $X$ possesses an $(n+1, s)$-disjoint and $c^{\prime} s$-bounded cover for every $s>0$. 


\section{A simple Hurewicz-type theorem}

We now proceed to the aforementioned version of the Hurewicz theorem. We need the following lemma extracted from Theorem 2.4 in [8].

Lemma 1. Let $X$ be a metric space with an $(n+1, t)$-disjoint and D-bounded cover for some $n \geq 1$ and $t, D>0$. Then there exists an $\left(n+2, \frac{t}{3}\right)$-disjoint and $\left(D+\frac{2}{3} t\right)$-bounded cover of $X$ with each point $x \in X$ belonging to at least two sets of different colours.

Proof. By assumption, $X$ has an $(n+1, t)$-disjoint and $D$-bounded cover $\mathscr{C}=$ $\bigcup_{k=1}^{n+1} \mathscr{C}_{k}$. For $C \in \mathscr{C}$, let $C^{\prime}$ denote the closed $\frac{t}{3}$-neighbourhood of $C$. Define a new cover $\bigcup_{k=1}^{n+1} \mathscr{C}_{k}^{\prime}$, where $\mathscr{C}_{k}^{\prime}:=\left\{C^{\prime}: C \in \mathscr{C}_{k}\right\}$. Notice that this cover is $\left(n+1, \frac{t}{3}\right)$-disjoint and $\left(D+\frac{2}{3} t\right)$-bounded. Now define sets of an additional colour as follows. For every $B \in \mathscr{C}$ of colour $j$, let $B^{0}$ be the set $B$ minus the union of all $C^{\prime} \in \bigcup_{k \neq j} \mathscr{C}_{k}^{\prime}$, and put $\mathscr{C}_{n+2}^{\prime}:=\left\{B^{0}: B \in \mathscr{C}\right\}$. The cover $\mathscr{C}^{\prime}:=\bigcup_{k=1}^{n+2} \mathscr{C}_{k}^{\prime}$ will have the required properties.

Clearly, $\mathscr{C}^{\prime}$ is still $\left(D+\frac{2}{3} t\right)$-bounded, as $\operatorname{diam}\left(B^{0}\right) \leq \operatorname{diam}(B) \leq D$ for all $B \in \mathscr{C}$. To verify that $\mathscr{C}^{\prime}$ is $\left(n+2, \frac{t}{3}\right)$-disjoint, it remains to check that $d\left(A^{0}, B^{0}\right) \geq \frac{t}{3}$ whenever $A \in \mathscr{C}_{i}, B \in \mathscr{C}_{j}$, and $A \neq B$. If $i=j$, then $d\left(A^{0}, B^{0}\right) \geq d(A, B) \geq t$, because $\mathscr{C}_{i}$ is $t$-disjoint. If $i \neq j$, then $A^{0} \subset A$ and $B^{0} \subset B \backslash A^{\prime}$ by construction of $\mathscr{C}_{n+2}^{\prime}$, thus $d\left(A^{0}, B^{0}\right) \geq d\left(A, B \backslash A^{\prime}\right) \geq \frac{t}{3}$. Now let $x \in X$. There exist $j \in$ $\{1, \ldots, n+1\}$ and $B \in \mathscr{C}_{j}$ such that $x \in B$. If $x$ belongs to some set $C^{\prime} \in \mathscr{C}_{k}^{\prime}$ with $k \neq j$, then $x \in B^{\prime} \cap C^{\prime}$. If there is no such set $C^{\prime}$ containing $x$, then $x \in B^{\prime} \cap B^{0}$. Thus in either case, $x$ belongs to at least two sets of different colours.

The following result corresponds to Theorem 7.2 in [8] for the case of a Lipschitz function $f: X \rightarrow \mathbb{R}$. The argument can also easily be adapted to the asymptotic dimension (see Theorem 1 in [3] for an earlier result in this direction).

Theorem 1. Let $X$ be a metric space, and let $f: X \rightarrow \mathbb{R}$ be a 1-Lipschitz function. Suppose that there exist $n \geq 1$ and $c>0$ such that for all $r \in \mathbb{R}$ and $t>0$, the set $f^{-1}([r, r+t))$ possesses an $(n+1, t)$-disjoint and $c t$-bounded cover. Then $X$ has Nagata dimension at most $n+1$ with a constant depending only on $n$ and $c$.

Proof. Fix $s>0$ and let $t:=(n+2) s$. For every $k \in \mathbb{Z}$, put $I_{k}:=[k t,(k+1) t)$.

For every odd integer $k$, proceed with the following construction. By assumption, $f^{-1}\left(I_{k}\right)$ admits an $(n+1, t)$-disjoint and $c t$-bounded cover. Lemma 1 now provides an $\left(n+2, \frac{t}{3}\right)$-disjoint and $(c+1) t$-bounded cover $\mathscr{C}_{k}=\bigcup_{i=1}^{n+2} \mathscr{C}_{k}^{i}$ with each point $x \in f^{-1}\left(I_{k}\right)$ belonging to two sets of different colours. Let $\mathscr{B}_{k}=\bigcup_{i=1}^{n+2} \mathscr{B}_{k}^{i}$ be the $(n+2, s)$-disjoint cover of $I_{k}$ such that $\mathscr{B}_{k}^{i}$ consists of the connected components of $I_{k} \backslash[k t+(i-1) s, k t+i s)$. Every point of $I_{k}$ is in $n+1$ sets of pairwise different colours. For $i=1, \ldots, n+2$, define

$$
\mathscr{D}_{k}^{i}:=\left\{C \cap f^{-1}(B): C \in \mathscr{C}_{k}^{i}, B \in \mathscr{B}_{k}^{i}\right\} .
$$

Note that if $x \in f^{-1}\left(I_{k}\right)$, then $x$ belongs to two sets of different colours of $\mathscr{C}_{k}$, and $f(x)$ is in $n+1$ sets of pairwise different colours of $\mathscr{B}_{k}$; thus there exists an index $i \in\{1, \ldots, n+2\}$ such that $x$ belongs to some set $C \cap f^{-1}(B) \in \mathscr{D}_{k}^{i}$. Hence, $\bigcup_{i=1}^{n+2} \mathscr{D}_{k}^{i}$ is a covering of $f^{-1}\left(I_{k}\right)$. Since $\mathscr{C}_{k}$ is $(c+1) t$-bounded, this cover is $c^{\prime} s$-bounded for $c^{\prime}:=(n+2)(c+1)$, and since $s \leq \frac{t}{3}$ and $f$ is 1 -Lipschitz, $\mathscr{D}_{k}^{i}$ is $s$-disjoint. For $i \in\{1, \ldots, n+2\}$, let $\mathscr{D}^{i}:=\bigcup_{k \text { odd }} \mathscr{D}_{k}^{i}$. Note that $\mathscr{D}^{i}$ is still $s$-disjoint.

Now put $s^{\prime}:=\left(c^{\prime}+2\right) s$ and note that $s^{\prime} \geq(n+2) s=t$. For every even integer $k$, there exists by assumption an $\left(n+1, s^{\prime}\right)$-disjoint and $c s^{\prime}$-bounded cover $\bigcup_{i=1}^{n+1} \mathscr{E}_{k}^{i}$ of 
$f^{-1}\left(I_{k}\right)$ by subsets of $f^{-1}\left(I_{k}\right)$. For $i \in\{1, \ldots, n+1\}$, let $\mathscr{E}^{i}:=\bigcup_{k \text { even }} \mathscr{E}_{k}^{i}$. The union

$$
\bigcup_{i=1}^{n+1}\left(\mathscr{D}^{i} \cup \mathscr{E}^{i}\right) \cup \mathscr{D}^{n+2}
$$

is an $(n+2)$-coloured covering of $X$ which we shall modify to satisfy the required properties. For every $E \in \mathscr{E}^{i}$, let $E^{*}$ be the union of $E$ with all sets of $\mathscr{D}^{i}$ at distance $<s$ from $E$. Clearly $\operatorname{diam}\left(E^{*}\right) \leq c s^{\prime}+2\left(c^{\prime}+1\right) s=c^{\prime \prime} s$ for some $c^{\prime \prime}=c^{\prime \prime}(n, c)$. We claim that $d\left(E^{*}, F^{*}\right) \geq s$ whenever $E, F \in \mathscr{E}^{i}$ are distinct. If $E$ and $F$ belong to the same family $\mathscr{E}_{k}^{i}$, this holds since $d(E, F) \geq s^{\prime} \geq 2 s+\operatorname{diam}(D)$ for all $D \in \mathscr{D}^{i}$ and $\mathscr{D}^{i}$ is $s$-disjoint. In the other case, $d(E, F) \geq t \geq s$, and by construction no set $D \in \mathscr{D}^{i}$ is at distance $<s$ from both $E$ and $F$, so the claim follows again since $\mathscr{D}^{i}$ is $s$-disjoint. In the final covering, the collection of sets of colour $i$ consists of all $E^{*}$ with $E \in \mathscr{E}^{i}$ and the remaining elements of $\mathscr{D}^{i}$ not belonging to such an $E^{*}$. This gives an $(n+2, s)$-disjoint and $c^{\prime \prime} s$-bounded cover of $X$. Since $s>0$ was arbitrary, $\operatorname{dim}_{\mathrm{N}}(X) \leq n+1$.

\section{Planar metric spaces}

We now turn to planar geodesic spaces. The proof of Theorem 2 below relies on the following result from [10].

Proposition 2. There is a universal constant $c_{1}$ such that the following holds. Suppose that $X$ is a planar geodesic metric space or a planar graph, and let $z \in X$ be a base point. Then for any $r, s>0$, the metric annulus $\{x \in X: r \leq d(z, x) \leq r+s\}$ admits a $c_{1} s$-bounded cover of s-multiplicity at most 2 .

See Lemma 4.4 in [10] for $M=10 \mathrm{~m}$. The very generous estimates show that one can take $c_{1}=10^{6}$. A somewhat modified and optimized argument yielding the constant $c_{1}=39$ has been worked out in [13].

Theorem 2. There is a universal constant $c_{2}$ such that every planar geodesic metric space or planar graph has Nagata dimension at most 2 with constant $c_{2}$.

Proof. Let $X$ be a planar geodesic metric space or a planar graph, let $z \in X$ be a base point, and put $f:=d(z, \cdot)$. By Proposition 2 , for any $r, s>0$, the set $f^{-1}([r, r+s])$ admits a $c_{1} s$-bounded cover of $s$-multiplicity at most 2 . It follows from Proposition 1 that $f^{-1}([r, r+s])$ possesses a $(2, \lambda s)$-disjoint and $c_{1}^{\prime} \lambda s$-bounded cover, where $\lambda$ and $c_{1}^{\prime}$ depend only on $c_{1}$. If $\lambda<1$, put $t:=\lambda s$ and $c:=c_{1}^{\prime}$. If $\lambda \geq 1$, put $t:=s$ and $c:=c_{1}^{\prime} \lambda$. In either case, for any $r, t>0$, the set $f^{-1}([r, r+t])$ has a $(2, t)$-disjoint and $c t$-bounded cover. Now the result follows from Theorem 1 .

\section{Three-dimensional Hadamard manifolds}

We proceed to Hadamard manifolds, that is, complete and simply connected Riemannian manifolds of non-positive sectional curvature.

Theorem 3. There is a universal constant $c_{3}$ such that every 3-dimensional Hadamard manifold has Nagata dimension 3 with constant $c_{3}$.

Proof. Let $(Y, d)$ be a 3-dimensional Hadamard manifold. Fix a Busemann function $f: Y \rightarrow \mathbb{R}$. Recall that $f$ is 1-Lipschitz, all horoballs $f^{-1}((-\infty, s])$ are convex, and the nearest point retraction from $Y$ onto any horoball is 1-Lipschitz. Furthermore, every horosphere $f^{-1}\{s\}$ is homeomorphic to $\mathbb{R}^{2}$, and the induced inner 
metric on $f^{-1}\{s\}$ is finite and complete, hence geodesic. (See [2]. By Proposition 3.1 in [12], Busemann functions and horospheres are in fact $C^{2}$, but this is not needed here.) Now fix $r \in \mathbb{R}$ and $t>0$. Put $A:=f^{-1}\left(\left[r-\frac{t}{2}, r+t\right]\right)$ and $H:=f^{-1}\left\{r-\frac{t}{2}\right\}$. The nearest point retraction $\pi: A \rightarrow H$ is 1-Lipschitz with respect to $d$ and hence does not increase the length of curves. Therefore, if $d_{A}$ and $d_{H}$ denote the induced inner metrics on $A$ and $H$, then $\pi:\left(A, d_{A}\right) \rightarrow\left(H, d_{H}\right)$ is still 1-Lipschitz. By Theorem 2, $\left(H, d_{H}\right)$ has Nagata dimension at most 2 with constant $c_{2}$, thus by Proposition 1 there is a universal constant $c_{2}^{\prime}$ such that $\left(H, d_{H}\right)$ possesses a $(3, t)$-disjoint and $c_{2}^{\prime} t$ bounded cover $\mathscr{B}=\bigcup_{i=1}^{3} \mathscr{B}_{i}$ by subsets of $H$. The sets $\pi^{-1}(B)$ with $B \in \mathscr{B}$ form a $(3, t)$-disjoint and $\left(c_{2}^{\prime}+3\right) t$-bounded cover of $\left(A, d_{A}\right)$. Furthermore, by taking the intersections with $A^{\prime}:=f^{-1}([r, r+t])$, we get a cover of $A^{\prime}$ that is $(3, t)$-disjoint and $\left(c_{2}^{\prime}+3\right) t$-bounded with respect to $d$, because $d\left(y, y^{\prime}\right) \leq d_{A}\left(y, y^{\prime}\right)$ for all $y, y^{\prime} \in A^{\prime}$, with equality if $d\left(y, y^{\prime}\right) \leq t$. Since $r \in \mathbb{R}$ and $t>0$ were arbitrary, it follows from Theorem 1 that $Y$ has Nagata dimension at most 3 with a universal constant $c_{3}$. In fact, $\operatorname{dim}_{\mathrm{N}}(Y)=3$, as $Y$ is locally bi-Lipschitz homeomorphic to $\mathbb{R}^{3}$.

Finally, we deduce a new Lipschitz extension result. Recall that a metric space $Y$ is an absolute $C$-Lipschitz retract, for a constant $C \geq 1$, if for every isometric inclusion $Y \subset X$ into another metric space $X$ there is a $C$-Lipschitz retraction of $X$ onto $Y$. Equivalently, for every metric space $X$ and every $\lambda$-Lipschitz map $f: D \rightarrow Y$, where $\lambda>0$ and $D \subset X$, there exists a $C \lambda$-Lipschitz extension $\bar{f}: X \rightarrow Y$ of $f$ (see Proposition 1.2 in [5]). It was shown in [15] that Hadamard manifolds with pinched negative sectional curvature, homogeneous Hadamard manifolds, and all 2dimensional Hadamard manifolds are absolute Lipschitz retracts (in the last case, one can take $C=4 \sqrt{2}$ ). Furthermore, by [9], the universal cover of any closed Riemannian 3-manifold of non-positive curvature is an absolute Lipschitz retract. We can now settle the 3-dimensional case completely.

Theorem 4. There is a universal constant $c_{4}$ such that every 3-dimensional Hadamard manifold is an absolute $c_{4}$-Lipschitz retract.

Proof. Corollary 1.8 in [16] states that a metric space $Y$ with $\operatorname{dim}_{N}(Y) \leq n$ is an absolute Lipschitz retract if and only if $Y$ is complete and Lipschitz $n$-connected. This latter condition means that there is a constant $\gamma \geq 1$ such that for $m=0,1, \ldots, n$, every $\lambda$-Lipschitz map from the unit sphere $S^{m} \subset \mathbb{R}^{m+1}$ into $Y$ can be extended to a $\gamma \lambda$-Lipschitz on the unit ball $B^{m+1} \subset \mathbb{R}^{m+1}$. The proof (using Theorem 1.6 in [16]) shows in fact that if a complete metric space $Y$ is Lipschitz $n$-connected with constant $\gamma$ and satisfies $\operatorname{dim}_{\mathrm{N}}(Y) \leq n$ with constant $c$, then $Y$ is an absolute $C$-Lipschitz retract for some $C=C(n, \gamma, c)$. Now if $Y$ is a Hadamard manifold (of any dimension), then the distance function on $Y$ is convex (see [2]), and it follows readily that $Y$ is Lipschitz $n$-connected for all $n$ with an absolute constant $\gamma$ (see, for example, Proposition 6.2.2 in [17]). By virtue of Theorem 3, this gives the result.

It remains an open question whether every Hadamard manifold of dimension bigger than 3, without extra conditions, has finite Nagata dimension and is therefore an absolute Lipschitz retract. 


\section{References}

[1] Assouad, P.: Sur la distance de Nagata. - C. R. Acad. Sci. Paris Sér. I Math. 294:1, 1982, $31-34$.

[2] Ballmann, W., M. Gromov, and V. Schroeder: Manifolds of nonpositive curvature. Progr. Math. 61, Birkhäuser, 1985.

[3] Bell, G. C., and A. N. Dranishnikov: A Hurewicz-type theorem for asymptotic dimension and applications to geometric group theory. - Trans. Amer. Math. Soc. 358:11, 2006, 4749-4764.

[4] Bell, G., and A. Dranishnikov: Asymptotic dimension in Będlewo. - Topology Proc. 38, 2011, 209-236.

[5] Benyamini, Y., and J. Lindenstrauss: Geometric nonlinear functional analysis. Volume 1. - Amer. Math. Soc. Coll. Publ. 48, Amer. Math. Soc., 2000.

[6] Bonamy, M., N. Bousquet, L. Esperet, C. Groenland, F. Pirot, and A. Scott: Surfaces have (asymptotic) dimension 2. - arXiv:2007.03582 [math.CO].

[7] Bonamy, M., N. Bousquet, L. Esperet, C. Groenland, C.-H. Liu, F. Pirot, and A. Sсотт: Asymptotic dimension of minor-closed families and Assouad-Nagata dimension of surfaces. - arXiv:2012.02435 [math.CO].

[8] Brodskiy, N., J. Dydak, M. Levin, and A. Mitra: A Hurewicz theorem for the AssouadNagata dimension. - J. Lond. Math. Soc. (2) 77:3, 2008, 741-756.

[9] Buyalo, S., and V. Schroeder: Extension of Lipschitz maps into 3-manifolds. - Asian J. Math. 5:4, 2001, 685-704.

[10] Fujiwara, K., and P. Papasoglu: Asymptotic dimension of planes and planar graphs. Trans. Amer. Math. Soc. 374:12, 2021, 8887-8901.

[11] Gromov, M.: Asymptotic invariants of infinite groups. - In: Geometric group theory, vol. 2 (Sussex, 1991), London Math. Soc. Lecture Note Ser. 182, Cambridge Univ. Press, 1993, 1-295.

[12] Heintze, E., and H.-C. Im Hof: Geometry of horospheres. - J. Differential Geom. 12:4, 1977, 481-491.

[13] Jørgensen, M.: Geodesic spaces of low Nagata dimension. - Master's thesis, ETH Zürich, 2020.

[14] Jørgensen, M., and U. LAng: Geodesic spaces of low Nagata dimension. - arXiv: 2004.10576v1 [math.MG].

[15] Lang, U., B. Pavlović, and V. Schroeder: Extensions of Lipschitz maps into Hadamard spaces. - Geom. Funct. Anal. 10:6, 2000, 1527-1553.

[16] Lang, U., and T. Schlichenmaier: Nagata dimension, quasisymmetric embeddings, and Lipschitz extensions. - Int. Math. Res. Not. IMRN 2005:58, 2005, 3625-3655.

[17] Schlichenmaier, T.: A quasi-symmetrically invariant notion of dimension and absolute Lipschitz retracts. - Diss. ETH No. 16216, ETH Zürich, 2005.

Received 22 December 2020 • Accepted 29 March 2021 • Published online 30 November 2021

Martina Jørgensen
ETH Zürich
Department of Mathematics
Rämistrasse 101
8092 Zürich
Switzerland
martina.joergensen@math.ethz.ch
Urs Lang

ETH Zürich

Department of Mathematics

Rämistrasse 101

8092 Zürich

Switzerland

lang@math.ethz.ch 